\title{
Early-branching euteleost relationships: Areas of congruence between concatenation and coalescent model inferences
}

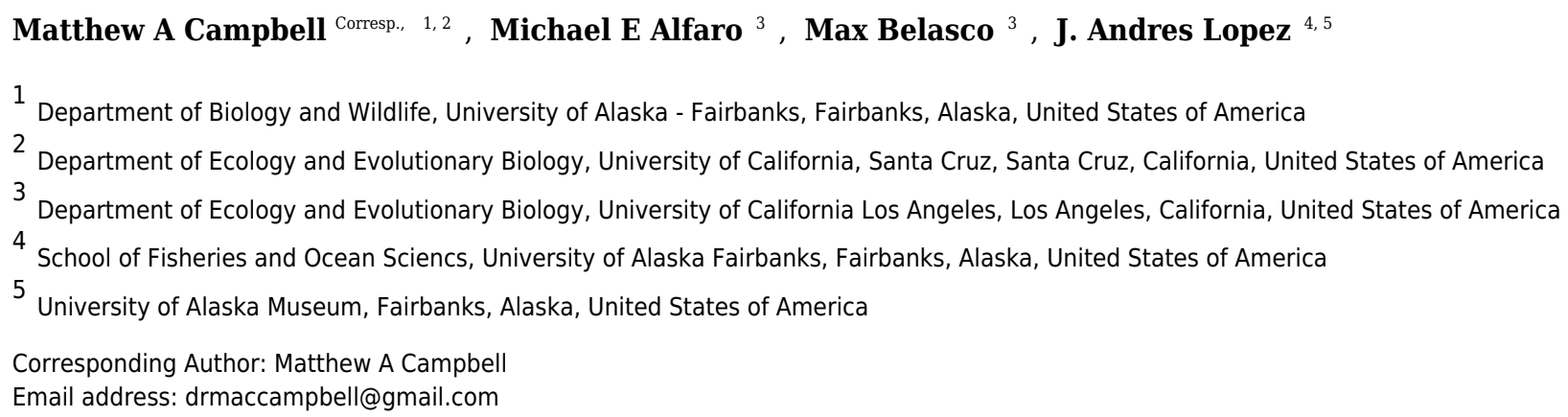

Phylogenetic inference based on evidence from DNA sequences has led to significant strides in the development of a stable and robustly supported framework for the vertebrate tree of life. To date, the bulk of those advances have relied on sequence data from a small number of genome regions that have proven unable to produce satisfactory answers to consistently recalcitrant phylogenetic questions. Here, we re-examine phylogenetic relationships among early-branching euteleostean fish lineages classically grouped in the Protacanthopterygii using DNA sequence data surrounding ultraconserved elements. We report and examine a dataset of thirty-four OTUs with 17,957 aligned characters from fifty-three nuclear loci. Phylogenetic analysis is conducted in concatenated, joint gene trees and species tree estimation and summary coalescence frameworks. All analytical frameworks yield supporting evidence for existing hypotheses of relationship for the placement of Lepidogalaxias salamandroides, monophyly of the Stomiatii and the presence of an esociform + salmonid clade. Lepidogalaxias salamandroides and the Esociformes + Salmoniformes are successive sister lineages to all other euteleosts in the majority of analyses. The concatenated and joint gene trees and species tree analysis types receiving high support values for this arrangement. However, inter-relationships of Argentiniformes, Stomiatii and Neoteleostei remain uncertain as they varied by analysis type while receiving strong and contradictory indices of support. Topological differences between analysis types are apparent within the otomorph and the percomorph taxa in the data set. Our results identify concordant areas with strong support for relationships within and between early-branching euteleost lineages but they also reveal limitations in the ability of larger datasets to conclusively resolve other aspects of that phylogeny. 
1 Title: Early-branching euteleost relationships: Areas of congruence between concatenation and

2 coalescent model inferences

3

4 Authors and Affiliations:

5 Matthew A. Campbell ${ }^{\mathrm{a}, 1^{*}}$, Michael E. Alfaro ${ }^{\mathrm{b}}$, Max Belasco ${ }^{\mathrm{b}}, \mathrm{J}$. Andrés López ${ }^{\mathrm{c}, \mathrm{d}}$

$6 \quad$ a Department of Biology and Wildlife, University of Alaska Fairbanks, Fairbanks, AK 99775

7 USA

$8 \quad{ }^{b}$ Department of Ecology and Evolutionary Biology, University of California Los Angeles, Los

9 Angeles, CA 90095 USA

10 c School of Fisheries and Ocean Sciences, University of Alaska Fairbanks, Fairbanks, AK 99775,

11 USA

12 d University of Alaska Museum, Fairbanks, AK 99775, USA

13

14 *Corresponding Author: DrMacCampbell@gmail.com

$15{ }^{1}$ Present Address: Department of Ecology and Evolutionary Biology, University of California

16 Santa Cruz, Santa Cruz, CA 95064, USA 


\section{Abstract}

18 Phylogenetic inference based on evidence from DNA sequences has led to significant strides in

19 the development of a stable and robustly supported framework for the vertebrate tree of life. To

20 date, the bulk of those advances have relied on sequence data from a small number of genome

21 regions that have proven unable to produce satisfactory answers to consistently recalcitrant

22 phylogenetic questions. Here, we re-examine phylogenetic relationships among early-branching

23 euteleostean fish lineages classically grouped in the Protacanthopterygii using DNA sequence

24 data surrounding ultraconserved elements. We report and examine a dataset of thirty-four OTUs

25 with 17,957 aligned characters from fifty-three nuclear loci. Phylogenetic analysis is conducted

26 in concatenated, joint gene trees and species tree estimation and summary coalescence

27 frameworks. All analytical frameworks yield supporting evidence for existing hypotheses of

28 relationship for the placement of Lepidogalaxias salamandroides, monophyly of the Stomiatii

29 and the presence of an esociform + salmonid clade. Lepidogalaxias salamandroides and the

30 Esociformes + Salmoniformes are successive sister lineages to all other euteleosts in the majority

31 of analyses. The concatenated and joint gene trees and species tree analysis types receiving high

32 support values for this arrangement. However, inter-relationships of Argentiniformes, Stomiatii

33 and Neoteleostei remain uncertain as they varied by analysis type while receiving strong and

34 contradictory indices of support. Topological differences between analysis types are apparent

35 within the otomorph and the percomorph taxa in the data set. Our results identify concordant

36 areas with strong support for relationships within and between early-branching euteleost lineages

37 but they also reveal limitations in the ability of larger datasets to conclusively resolve other

38 aspects of that phylogeny. 


\section{Introduction}

40 Phylogenomic datasets comprising hundreds to thousands of genome segments produced through

41 high throughput sequencing technology have shown promise to resolve difficult phylogenetic

42 problems (e.g. Faircloth et al., 2013, 2012; Gilbert et al., 2015; Harrington et al., 2016; Lemmon

43 and Lemmon, 2013). At the same time, novel and refined inference tools including

44 implementations of the multispecies coalescent model to address incomplete lineage sorting

45 (ILS) through Gene Trees-to-Species Tree (GT-ST) methods (Knowles and Kubatko, 2011)

46 continue to extend the power and complexity of phylogenetic research. Despite these advances in

47 genomic-scale dataset production and phylogenetic inference, difficult areas of the tree of life

remain unresolved (Delsuc et al., 2005; Pyron, 2015; Rokas and Carroll, 2006). Relationships

among early-branching euteleost lineages remain nebulous (e.g. Betancur-R. et al., 2013;

Campbell et al., 2013; Li et al., 2010; Near et al., 2012) and stand out as one of the most

contentious regions of the fish tree of life. Although this question has been studied from

morphological and molecular perspectives consensus has yet to emerge.

The name Euteleostei was first applied to a diverse group of fishes that includes all teleosts outside of the superorders Elopomorpha, Osteoglossomorpha and Clupeomorpha by phyletic analysis (Greenwood et al., 1967, 1966). Rosen (1985) excluded esocoids from the Euteleostei based on cladistic analyses of morphological characters, while Johnson and Patterson (1996) included esocoids but excluded ostariophysans. Subsequent phylogenetic studies of mitochondrial (e.g. López et al. 2004; Lavoué et al., 2008) and nuclear DNA (e.g. Betancur-R. et al., 2013; Near et al., 2012) supported a monophyletic Euteleostei including esocoids but excluding Ostariophysi and the Alepocephaliformes (previously classified in Argentiniformes

61 nested in the Euteleostei). 
Recent phylogenetic studies based on molecular evidence consistently support the

63

monophyly of five major euteleost lineages (Betancur-R. et al., 2013; Campbell et al., 2013; Li et al., 2010; Near et al., 2012): 1) a clade formed by Esociformes and Salmoniformes; 2) the Stomiatii sensu Betancur-R. et al. (2013) consisting of Osmeriformes (excluding Galaxiiformes) and Stomiiformes; 3) the Argentiniformes (excluding the Alepocephaliformes); 4) the Galaxiiformes (excluding Lepidogalaxias); and 5) the Neoteleostei. In addition, these studies agree on placing the monotypic Lepidogalaxias as the sister group of all other euteleosts. Aside from the placement of Lepidogalaxias, there is little congruence among different studies regarding relationships among the five lineages (e.g. Betancur-R. et al., 2013; Campbell et al., 2013; Li et al., 2010; Near et al., 2012). The early branching patterns of euteleosts are still in need of further study and represent a difficult problem for traditional morphological and molecular phylogenetics.

Here we apply the "new and general theory of molecular systematics" (Edwards, 2009) to examine early-branching euteleost relationships using multi-locus datasets generated by targeted enrichment of conserved nuclear DNA sequences. Concatenated and GT-ST phylogenetic inference frameworks are used to assess the stability and strength of evidence for alternative arrangements in this poorly resolved section of the fish tree of life.

\section{Material and Methods}

\subsection{Taxon and character sampling}

We targeted species representing five of the six major euteleost lineages as well as several noneuteleost outgroups (Supplementary Table S1). We prepared genomic DNA libraries with 500$600 \mathrm{bp}$ inserts by shearing total genomic DNA extracts to size using a sonicator (Diagenode, Inc) 
85 and ligating a set of custom-indexed Illumina Tru-Seq compatible adapters (Faircloth and Glenn,

86 2012) to the sheared DNA using reagents from a library preparation kit (KapaBiosystems, Inc.).

87 Adapter-ligated DNA was amplified with 16-18 cycles of PCR. To obtain sequences from

88 homologous loci across the taxonomic sample, we performed targeted enrichment of

89 ultraconserved element (UCEs) loci shared among acanthopterygians following protocols

90 outlined in Faircloth et al., (2013). We modified the capture protocol by pooling eight, indexed

91 sequencing libraries at equimolar ratios prior to enrichment and performing 12-16 cycles of

92 PCR-recovery after enrichment. Following the enrichment procedure, we quantified enriched,

93 amplified libraries using a commercial qPCR quantification kit (KapaBiosystems, Inc.), and we

94 prepared an equimolar pool of pooled libraries for sequencing on an Illumina HiSeq 2500

95 instrument using 100 base pair, paired-end sequencing chemistry in rapid run mode (UCLA

96 Neuroscience Genomics Core). To extend our taxon sampling, we included previously published

97 UCE data (Faircloth et al., 2013) in our analyses (Supplementary Table S1).

\subsection{Raw sequence data processing}

100 Demultiplexed reads were edited for length, overall quality and adapter contamination using

101 Trimmomatic v. 0.32 (Bolger et al., 2014). We assembled a subset of cleaned reads across

102 various kmers with Velvet v. 1.2.10 (Zerbino and Birney, 2008) to establish a range of suitable

103 kmers for assembly. We then assembled sequences for each species using two different

104 approaches. For non-salmonids, we assembled reads using VelvetOptimiser v. 2.2.5 across the

105 optimal range of kmers we identified (57 to 83). For salmonids, assemblies from Velvet were

106 produced for each value between 57 and 83 . However, as the optimization performed by

107 VelvetOptimiser is designed for haploid or diploid organisms, an alternative selection criterion 
108 of the maximum number of single copy UCE loci was chosen to accommodate the effect of

109 ancestral polyploidy in salmonid genomes (Allendorf and Thorgaard, 1984). A single dataset

110 assembly was retained downstream analyses from each alternative approach to data assembly.

111 We identified homologous UCE loci and prepared sequences for alignment with the PHYLUCE

112 pipeline (Faircloth, 2015). During orthology assessment, the PHYLUCE package screens for and

113 removes from analysis reciprocally duplicate enriched loci, which may represent paralogs.

\subsection{Alignment and phylogenetic analysis}

116 Following orthology assessment, the taxon set consisted of thirty-four Operational Taxonomic

117 Units (OTUs) representing outgroups and basal euteleost lineages. We ensured this taxon set

118 included loci sequenced in at least 31 of the 34 OTUs. We aligned data from all loci in with

119 MAFFT v. 7.130b (Katoh et al., 2002) through the PHYLUCE pipeline (Faircloth, 2015).

120 We analyzed the 34-OTU dataset under the Maximum-Likelihood (ML) framework as

121 implemented in RAxML v. 8.1.24 (Stamatakis, 2014). Each UCE locus was modeled as a

122 partition evolving under the general time reversible (GTR) model of sequence evolution with

123 gamma distributed rate variation $(\Gamma)$. We set ML pseudoreplicate searches to automatically stop

124 when stable bootstrap indices were detected (autoMRE). A joint gene trees and species tree

125 estimation was conducted in a Bayesian framework with *BEAST (Heled and Drummond, 2010)

126 as implemented in BEAST v. 2.1.3 (Drummond et al., 2012). We analyzed data using a constant

127 coalescent model under a Hasegawa-Kishino-Yano (HKY) model of sequence evolution with a

128 four-category gamma distributed rate variation $(\Gamma)$ and empirical base frequencies to each locus.

129 Convergence and sufficient effective sample sizes (ESSs, > 200) of all parameters were reached

130 by combining three chains of 800 Million generations with $40 \%$ burn-in. Two additional 
131 analyses were conducted. To verify that partitioning in the ML analysis by gene does not

132 influence early-branching euteleost relationships and support values, objective partitioning was

133 investigated. To verify that the use of the coalescent model in *BEAST resulted in an alternative

134 arrangement of early-branching euteleost lineages, not the choice of nucleotide evolution model,

135 a concatenated Bayesian analysis with BEAST 2 with the same nucleotide evolution model for

136 each UCE locus as *BEAST $(\mathrm{HKY}+\Gamma$ with empirical base frequencies) was undertaken

137 (Supplemental Document S1).

138

1392.3 Topology tests and occurrence of particular arrangements in the Bayesian tree posterior

140 sample

141 To determine the significance of UCE evidence corroborating or refuting alternative

142 phylogenetic arrangements, we tested the following topologies resulting from concatenated and

143 GT-ST analysis against each other: (1) the best-scoring ML topology; (2) the consensus species-

144 tree topology from *BEAST; and (3) a Protacanthopterygii sensu Betancur-R. et al. (2013) as the

145 sister lineage to the Stomiatii. A best scoring ML tree (1 from above) and constrained trees (2

146 and 3 from above) were generated with RAxML v. 8.2.3 partitioned by UCE using a GTR $+\Gamma$

147 model of nucleotide evolution. We tested the trees against each other by generating per site

148 likelihoods with RAxML and analyzing the output with CONSEL v. 0.20 (Shimodaira and

149 Hasegawa, 2001). CONSEL implements several hypothesis tests allowing a more rigorous

150 comparison between alternative hypotheses than solely comparing likelihood values.

151 As the *BEAST posterior tree presented as the consensus species-tree topology

152 represents the combination of many different species trees, we searched the combined post burn-

153 in posterior tree sample from the separate *BEAST chains $(180,003$ trees $)$ for alternative 
154 phylogenetic hypotheses to determine if the *BEAST algorithm considered these alternatives.

155 The *BEAST posterior tree sample was searched for the best scoring ML topology and a

156 monophyletic Protacanthopterygii sensu Betancur-R. et al. (2013) with Python scripts (Moravec,

157 2015).

158

1592.4 Summary coalescent analyses

160 To further examine the potential impacts of small regions in concatenated alignments on key

161 relationships differing between RAxML concatenated analyses and the *BEAST species tree, we

162 examined our data through summary coalescent analyses. These methods function on

163 independently estimated gene trees, therefore decreasing the influence of small regions in

164 concatenated analyses (Shen et al., 2017) and accounting for ILS. Furthermore, *BEAST and

165 BEAST assume a molecular clock that may contribute to discrepancies between these analyses

166 and RAxML analyses. For each of the UCE loci, we generated a gene tree with nucleotide

167 evolution modeled with the GTR nucleotide evolution model and gamma distributed rate

168 variation $(\Gamma)$ with RAxML version 8.0.19. A set of rooted gene trees were also generated by

169 specifying Polypterus senegalus as the outgroup. Four summary coalescent analysis frameworks

170 were applied to the gene trees. The Accurate Species TRee ALgorithm (ASTRAL) and Neighbor

171 Joining species tree (NJst) tree methods accept unrooted trees with missing taxa. The unrooted

172 gene trees were analyzed program ASTRAL version 4.10.12 (Mirarab et al., 2014; Mirarab et al.,

173 2015) and with NJst (Liu and Yu, 2011) via the Species TRee Analysis Web server (STRAW)

174 (Shaw et al., 2013). The rooted trees were analyzed with both STAR (Liu et al., 2009) and MP-

175 EST (Liu et al., 2010) through STRAW.

176 


\section{3. Results}

\section{3.1 Characteristics of UCE dataset}

179 Following orthology assessment and filtering for loci not present in 31 of 34 OTUs, the dataset is 180 composed of a total of 53 UCE loci, 17,957 characters, 9,576 distinct alignment patterns and

$18122.11 \%$ gaps or missing data. We present details of the number of UCE loci recovered for each

182 taxon, the average length of UCE matching contigs, average coverage of contigs matching UCEs

183 and number of duplicate loci removed in Supplementary Table S1. The assemblies and alignment

184 are available within the Data Supplement.

\subsection{Early-branching euteleost relationships}

187 Concatenated ML analysis supports a monophyletic Euteleostei, excluding Ostariophysi and 188 Alepocephaliformes (Bootstrap Support $[\mathrm{bs}]=100 \%$ ). Figure 1 shows the inferred branching 189 pattern among main euteleost groups from the 34-OTU dataset. Relationships among main 190 euteleost lineages in the concatenated ML topology are (Lepidogalaxias, ((Esociformes,

191 Salmoniformes), (Argentiniformes, (Stomiatii, Neoteleostei)))) with all nodes among those

192 lineages receiving strong support $(\mathrm{bs}=100 \%)$. GT-ST analysis of the dataset in *BEAST indicates a monophyletic Euteleostei with high

194 support, posterior probability $(\mathrm{pp})=1.00$ (Figure 2). A topology of (Lepidogalaxias,

195 ((Esociformes, Salmoniformes), ((Argentiniformes, Stomiatii), Neoteleostei))))) is generated in

196 this analysis. Support values for the placement of main euteleost lineages are high throughout the 197 consensus tree. The placement of Lepidogalaxias and the Esociformes + Salmoniformes receive 198 very high support $(\mathrm{pp}=1.00)$. Argentiniformes + Stomiatii as the sister lineage of the neoteolosts

199 received strong support $(\mathrm{pp}=0.99)$. A sister relationship between the Argentiniformes and 
200 Stomiatii was also well supported $(\mathrm{pp}=0.96)$. The GT-ST and ML inferred phylogenies differ

201 on the relationships among argentiniforms, stomiatians and neoteleosts.

202 Through the additional concatenated analyses presented in the Supplemental Document S1,

203 conflicts between ML and GT-ST results presented in Figures $1 \& 2$ are shown to be the product

204 of the distinct analytical frameworks and do not result from how data were modeled. The

205 additional concatenated analyses in Supplemental Document S1 show identical branching

206 patterns for main early-branching euteleost lineages to the concatenated ML analysis presented

207 in Figure 1 with high support values. Retaining the same model but changing the partitioning

208 strategy with RAxML demonstrates that the inferred phylogeny from the ML analysis presented

209 in Figure 1 is not sensitive to partitioning (Supplemental Figure S1). Not implementing a

210 *BEAST model, while retaining the same nucleotide evolution and partitioning scheme for a

211 concatenated analysis with BEAST 2 also produces a phylogeny (Supplemental Figure S2) with

212 the branching of main early-branching euteleost lineages matching that of the concatenated ML

213 analysis presented in Figure 1, not the *BEAST GT-ST analysis presented in Figure 2.

214 Consequently, the topological differences between phylogenies shown in Supplemental Figure

215 S2 and Figure 2 may be attributed to whether a concatenated or coalescent approach is

216 implemented. There are three key locations in the inferred trees that differ: (1) within the

217 Otomorpha, the placement of Bajacalifornia megalops, (2) the arrangement of early-branching

218 euteleost lineages, and (3) the arrangement of the three percomorph lineages. 


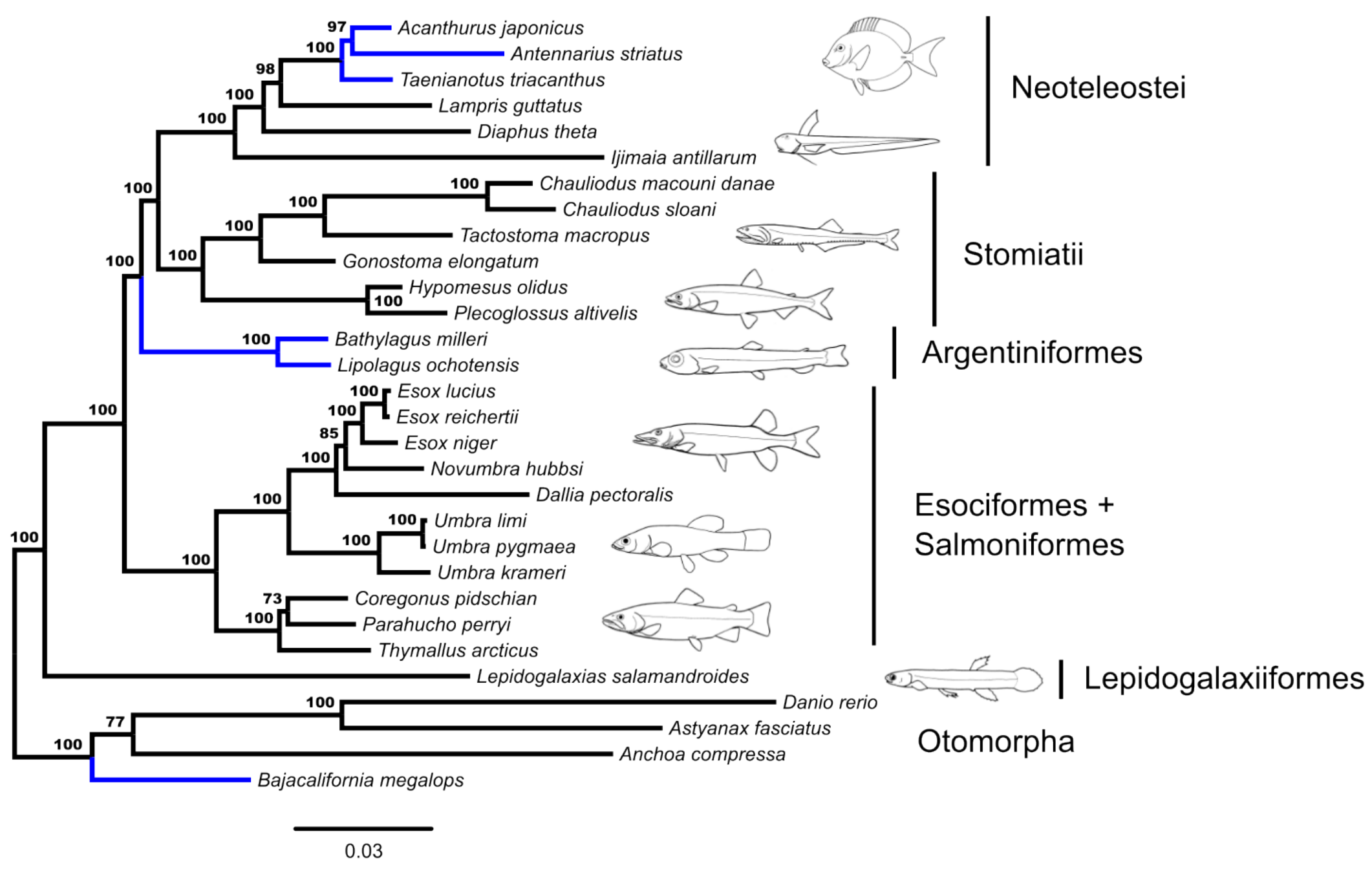


221 Figure 1: Phylogenetic tree from fifty-three ultraconserved element (UCE) loci generated in a concatenated framework with RAxML.

222 Each locus is designated as a partition and modeled under a GTR $+\Gamma$ model of nucleotide evolution. Values from automatic stopping

223 of bootstrap replicates are indicated at each node. The tree is rooted by Polypterus senegalus. Polypterus senegalus, Amia calva,

224 Osteoglossum bicirrhosum and Pantodon buchholzi are omitted from figure. Early-branching euteleost taxa are labeled and indicated 225 by drawings of a representative taxon (Nelson, 2006). From the Neoteleostei, Ateleopodiformes and Acanthuriformes drawings are 226 included. Placements of taxa that are different from the GT-ST topology (Figure 2) are indicated in blue. 


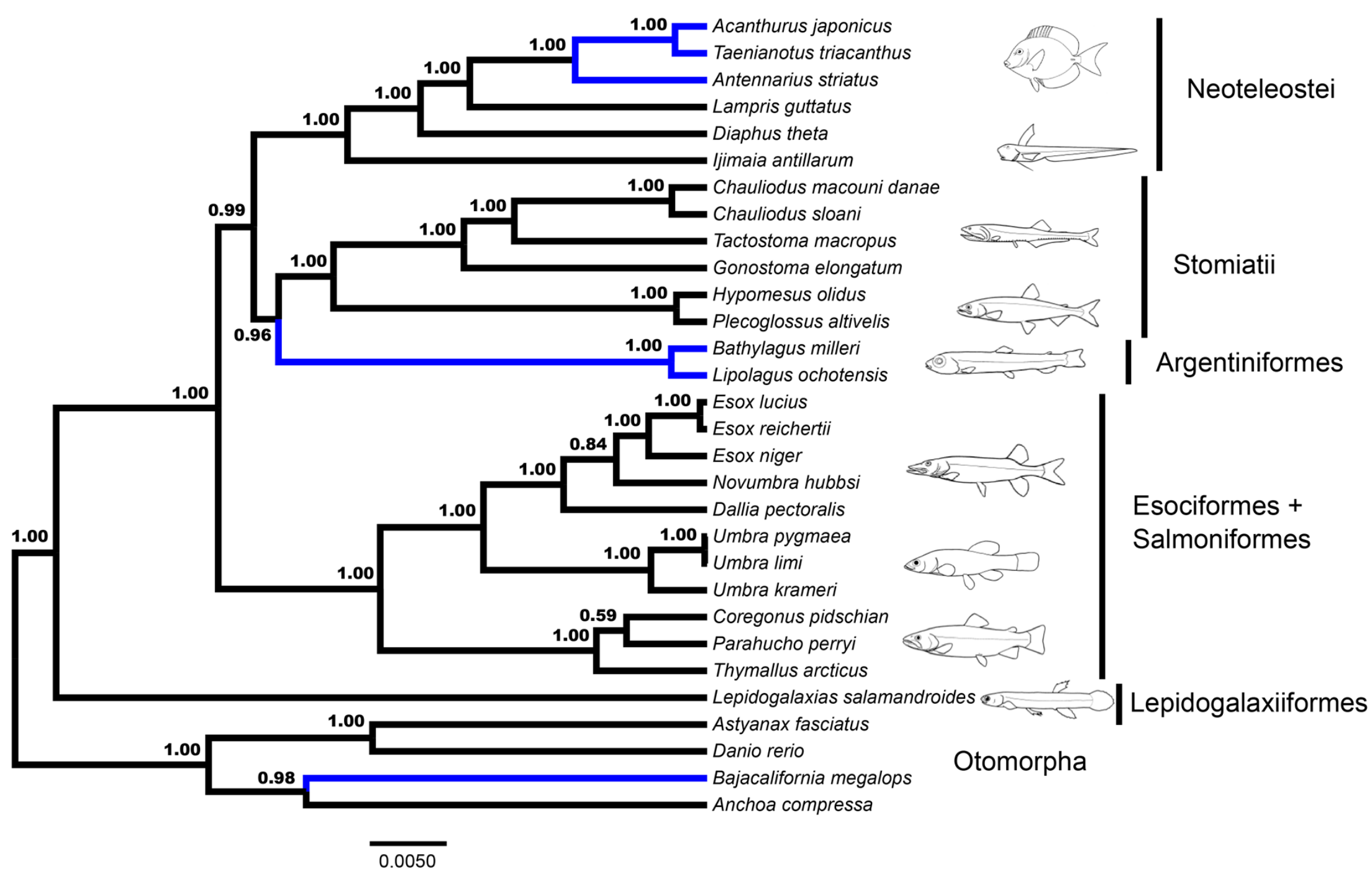


230 Figure 2: Species tree from *BEAST. Fifty-three ultraconserved element (UCE) loci are modeled under an HKY model of nucleotide

231 sequence evolution with a four category gamma distribution characterizing rate variation among sites $(\Gamma)$. Each model of sequence

232 evolution has independent model parameters. This tree represents the combination of three independent *BEAST runs with the

233 posterior probability of each node indicated. Early-branching euteleost lineages are labeled and indicated with representations of a

234 representative taxon (Nelson, 2006). Images of neoteleost lineages from Acanthuriformes and Ateleopodiformes are also included.

235 The tree is rooted by Polypterus senegalus. Polypterus senegalus, Amia calva, Osteoglossum bicirrhosum and Pantodon buchholzi are 236 omitted from figure. Placements of taxa that are different from the concatenated topology (Figure 1) are indicated in blue. 
2383.3 Topology tests and occurrence of particular arrangements in the Bayesian tree posterior

239 sample

240 Testing with CONSEL indicates the best-scoring ML tree, with a topology of (Lepidogalaxias,

241 ((Esociformes, Salmoniformes), (Argentiniformes, (Stomiatii, Neoteleostei)))), is significantly

242 better than the topology generated by GT-ST analysis with both the approximately unbiased test

$243\left(p=1 \times 10^{-5}\right)$ and the weighted Shimodaira-Hasegawa test $\left(p=1 \times 10^{-3}\right)$. A monophyletic

244 assemblage of protacanthopterygian taxa sensu Betancur-R. et al. (2013) sister to the Stomiatii is

245 significantly worse than the best-scoring ML tree with both the approximately unbiased test $(p=$

$\left.2468 \times 10^{-6}\right)$ and the weighted Shimodaira-Hasegawa test $\left(p=1 \times 10^{-4}\right)$. The posterior set of 180,003

247 trees generated by *BEAST did not include a single occurrence of either the ML best tree

248 topology or a monophyletic Protacanthopterygii sensu Betancur-R. et al. (2013).

$250 \quad 3.4$ Summary coalescent analyses

251 Fifty-three unrooted gene trees were analyzed by ASTRAL and NJst, the requirement of rooting

252 by Polypterus senegalus reduced the number of available gene trees that are rooted to forty-four

253 for STAR and MP-EST. The summary coalescent results vary with regards to the three key

254 differences identified previously as presented in Figures $1 \& 2$ (Table 1). ASTRAL (Figure 3)

255 and NJst resolves the otomorphs as fround by RAxML concatenation analyses, the early-

256 branching euteleost relationships as in RAxML and BEAST concatenation, while the

257 percomorph taxa are found in an arrangement from *BEAST and BEAST. ASTRAL posterior

258 probability values for the placement of the Argentiniformes and Stomiatii+Neoteleosteii are low

259 (0.58 and 0.56 respectively). STAR resolves the otomorphs and percomorphs as BEAST and

260 *BEAST analyses, but early-branching euteleosts match the concatenated RAxML results. MP- 
261 EST matches the concatenated RAxML results for otomorphs and BEAST / *BEAST for the 262 percomorphs while producing a unique topology of early-branching euteleost lineages. 
263 Table 1: Comparison of results from all phylogenetic analyses presented in this study of topological differences within the

264 Otomorpha, the early-branching euteleost lineages and the Percomorpha. Concatenation or Gene Trees-to-Species Tree (GT-ST)

265 framework and analysis program are indicated in the Method column. If a tree is presented, it is indicated in the Figure column. The

266 three key topological differences determined between concatenation in RAxML presented in Figure 1 and *BEAST presented in

267 Figure 2 are indicated and color-coded to analysis method. Results consistent with the RAxML results are blue and those consistent

268 with the *BEAST analysis are orange. A unique topology is not color-coded for early-branching euteleosts present only in the results

269 from MP-EST.

\begin{tabular}{|c|c|c|c|c|}
\hline \multirow[t]{2}{*}{ Method } & \multirow[t]{2}{*}{ Figure } & \multicolumn{3}{|l|}{ Area of Topological Difference } \\
\hline & & Otomorpha & Early-Branching Euteleosts & Percomorpha \\
\hline Concatenation RAxML & Figure 1 & (Bajacalifornia,(Anchoa,(Astyanax, Danio))) & (Lepidogalaxias,(Esociformes+Salmoniformes,(Argentiniformes,(Stomiatii, Neoteleosteii)))) & (Taenianotus, (Acanthurus, Antennarius )) \\
\hline GT-ST *BEAST & Figure 2 & $(($ Anchoa, Bajacalifornia $),($ Astyanax, Danio $))$ & (Lepidogalaxias, (Esociformes+Salmoniformes,((Argentiniformes,Stomiatii),(Neoteleosteii)))) & (Antennarius, (Acanthurus, Taenianotus)) \\
\hline Concatenation RAxML & Figure S1 & (Bajacalifornia,(Anchoa,(Astyanax, Danio))) & (Lepidogalaxias,(Esociformes+Salmoniformes,(Argentiniformes,(Stomiatii, Neoteleosteii)))) & (Taenianotus, (Acanthurus, Ante \\
\hline Concatenation BEAST & Figure S2 & $(($ Anchoa, Bajacalifornia $),($ Astyanax, Danio $))$ & (Lepidogalaxias, (Esociformes+Salmoniformes,(Argentiniformes,(Stomiatii, Neoteleosteii)))) & (Antennarius, (Acanthurus, Taenianotus)) \\
\hline GT-ST ASTRAL & Figure 3 & (Bajacalifornia, $($ Anchoa, $($ Astyanax, Danio $)))$ & (Lepidogalaxias,(Esociformes+Salmoniformes,(Argentiniformes,(Stomiatii, Neoteleostei & (Antennarius,(Acanthurus, Taenianotus)) \\
\hline GT-ST NJst & & (Bajacalifornia, $($ Anchoa, $($ Astyanax, Danio $)))$ & (Lepidogalaxias,(Esociformes+Salmoniformes,(Argentiniformes,(Stomiatii, Neoteleostei & (Antennarius, (Acanthurus, Taenianotus)) \\
\hline GT-ST STAR & & ((Anchoa, Bajacalifornia),(Astyanax, Danio $))$ & (Lepidogalaxias,(Esociformes+Salmoniformes,(Argentiniformes,(Stomiatii, Neoteleosteii)))) & (Antennarius,(Acanthurus, Taenianotus)) \\
\hline GT-ST MP-EST & & (Bajacalifornia,(Anchoa,(Astyanax, Danio))) & (Lepidogalaxias,(Argentiniformes,(Esociformes+Salmoniformes,(Stomiatii, Neoteleostei)))) & (Antennarius, (Acanthurus, Taenianotus)) \\
\hline
\end{tabular}




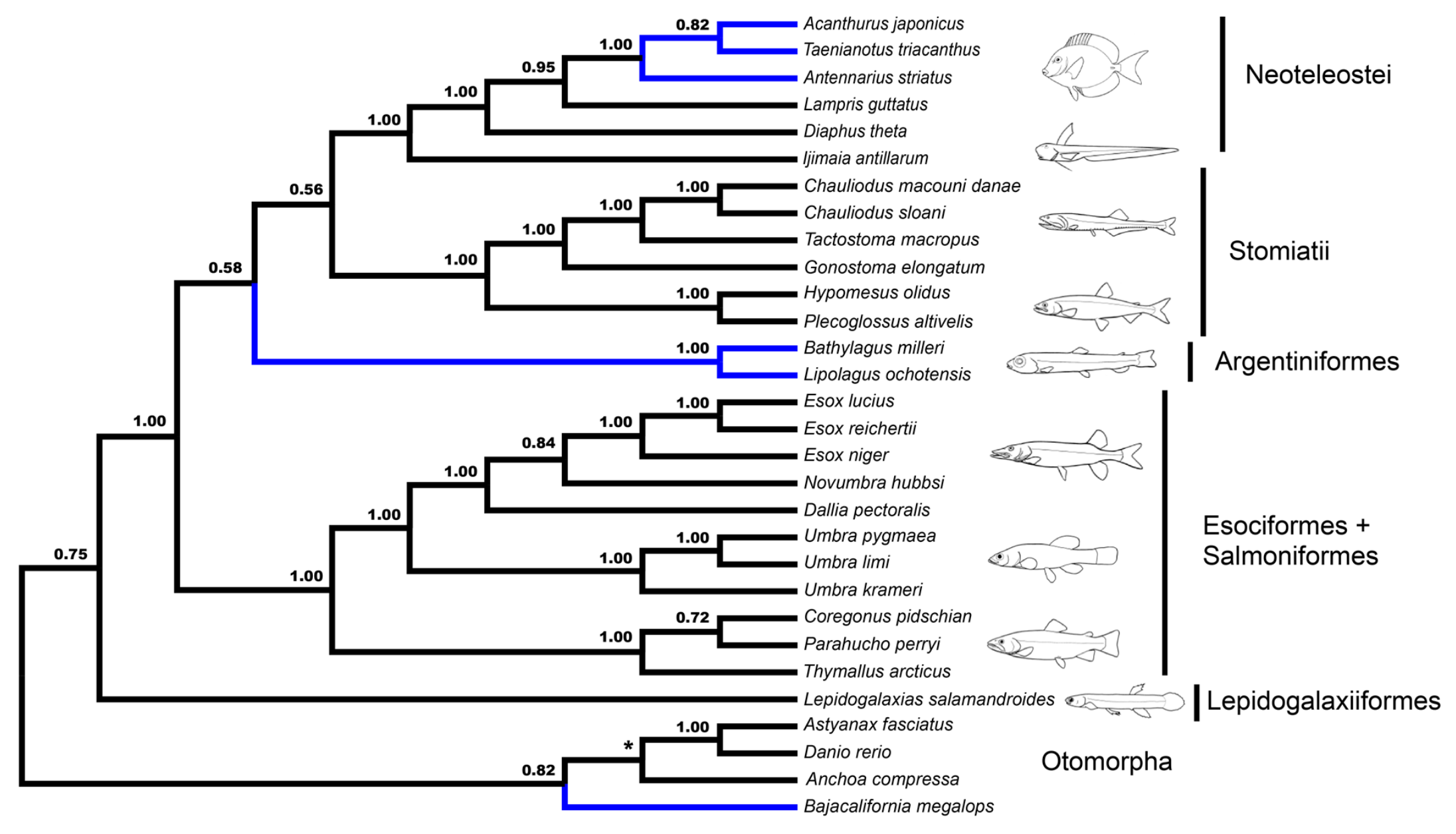

274 Figure 3: Species tree from ASTRAL. Gene trees from fifty-three ultraconserved element (UCE) loci were separately generated with

275 a GTR $+\Gamma$ model of nucleotide evolution with RAxML. All fifty-three gene trees were then processed by the ASTRAL algorithm

276 which rooted each tree arbitrarily with Anchoa compressa. Posterior probability for each node is indicated for all nodes except for the 
277 node involved with arbitrary rooting by ASTRAL for which no posterior probability was provided and is indicated with an asterisk

278 (*). Early-branching euteleost lineages are labeled and indicated with representations of a representative taxon (Nelson, 2006). Images 279 of neoteleost lineages from Acanthuriformes and Ateleopodiformes are also included. The species tree from ASTRAL was re-rooted

280 by Polypterus senegalus. Polypterus senegalus, Amia calva, Osteoglossum bicirrhosum and Pantodon buchholzi are omitted from

281 figure. Placement of lineages that were shown to be different in Figure 1 and Figure 2 are indicated in blue. 


\section{4. Discussion}

284 4.1 Hypotheses of early-branching euteleost relationships

285 Our phylogenomic analysis provides strong support for relationships of early diverging

286 euteleosts that consist of Lepidogalaxias and esociforms + salmoniforms as successive sister

287 lineages to a clade containing argentiniforms, stomiatiids and neoteleosts. Despite the most

288 intensive character sampling of this group to date, our analyses do not resolve two conflicting

289 hypotheses for relationships among the Argentiniformes, Stomiatii and Neoteleostei. The

290 concatenated ML derived topology resolves argentiniforms and stomatiids as successive sister

291 lineages to the neoteleosts as do most summary coalescent methods, while the *BEAST GT-ST

292 analysis recovers an argentiniform + stomiatiids clade as the sister group to neoteleosts.

293 Combined, our analyses yield strong support for the Esociformes + Salmoniformes clade,

294 which has found robust and consistent support in molecular phylogenetic studies (López et al.,

295 2004), reviewed by Campbell et al. (2013), despite weak or conflicting evidence from

296 morphology (Johnson and Patterson, 1996; Wilson and Williams, 2010). We also recover the

297 Stomiatii (Osmeriformes + Stomiiformes) with high support values in both analyses in this

298 study. On the other hand, we do not find a close relationship between the clade of Esociformes +

299 Salmoniformes and any other major group of early-branching euteleosts such as Argentiniformes

300 (Near et al., 2012). Instead, as shown in mitogenomic phylogenies (Campbell et al., 2013; Inoue

301 et al., 2003) or analyses of combined mitochondrial and nuclear data (Burridge et al., 2012), we

302 find Esociformes and Salmoniformes as sister to all other euteleosts in the study, with the

303 exclusion of Lepidogalaxias.

304

3054.2 Support for hypotheses of early-branching euteleost lineages 
306 Unlike other molecular (and morphological) studies of the euteleost phylogeny (e.g. Betancur-R.

307 et al., 2013; Li et al., 2010; Near et al., 2012), our conflicting topologies are strongly supported

308 by both bootstrap values and Bayesian posterior probabilities.

309 Earlier studies typically yield low or moderate support for relationships along this section of the

310 teleost phylogeny backbone. For example, the placement of the Argentiniformes and

311 Salmoniformes + Esociformes sister to the remaining three major euteleost lineages (Stomiati,

312 Galaxiiformes, and neoteleosts) receives a bootstrap support value between 70-89\% in Near et al.

313 (2012). Other nodes supporting the branching order of the five major euteleost lineages are

314 supported by $90-99 \%$ bootstrap support values. The bootstrap support for a sister

315 Protacanthopterygii sensu Betancur-R. et al. (2013) and Stomiatii is 36\%, and the monophyly of

316 Stomiatii receives a bootstrap support of $73 \%$ in the hypothesis presented by Betancur-R. et al.

317 (2013). In a mitochondrial genome based study, a sister relationship of Argentiniformes to the

318 Salmoniformes + Esociformes receives a bootstrap support of 74\% ( $\mathrm{Li}$ et al., 2010). In the same

319 study, the Argentiniformes, Salmoniformes and Esociformes are the sister lineage of the

320 Stomiatii, supported by an $81 \%$ bootstrap support value (Li et al., 2010).

321 While we find uncharacteristically high support for branching relationships among all of

322 the four major euteleost lineages represented in this study in a concatenated ML framework,

323 gauging the significance of high bootstrap values in analyses of large data matrices is

324 problematic. Bootstrap values may be high even with conflict or systematic error (Felsenstein,

325 1978; Hillis and Bull, 1993; Huelsenbeck, 1997). Concatenated ML phylogenomic analysis has

326 previously been demonstrated with 1,070 genes in yeasts to produce $100 \%$ bootstrap support for

327 all internodes, despite incorrect branching likely present (Salichos and Rokas, 2013). The

328 *BEAST GT-ST analysis also produces high support values; however, posterior probability 
329 values themselves are both conditioned on the model of evolution and are not guaranteed to have

330 good frequentist statistical behavior (Alfaro et al., 2003; Alfaro and Holder, 2006) and may be

331 misleading under certain conditions (Suzuki et al., 2002; Salichos and Rokas, 2013). Another

332 potential issue in phylogenetics is that a few loci may substantially influence results in

333 concatenated analyses (Shen et al., 2017). To mitigate the influence of small regions of large

334 effect in a concatenated analysis, we evaluated individual gene trees with four summary

335 coalescent analysis. Three of the four summary coalescent analyses were in agreement with the

336 RAxML concatenated analyses and the concatenated BEAST analysis hypothesis of early-

337 branching euteleost lineages. The MP-EST analysis produced a unique result regarding early-

338 branching euteleost lineages among all analyses. While the otomorph taxa are not consistently

339 found to have a certain topology in the summary coalescent methods, three of the four analyses

340 match the concatenated RAxML results. The summary coalescent methods indicate that the

341 early-branching euteleost topology of *BEAST may be a result of the underlying assumption of a

342 molecular clock in this analysis or other issue. The consistent results for percomorph

343 relationships across GT-ST methods suggests that a particular locus or subset of loci and/or

344 incomplete lineage sorting is affecting the concatenated analysis in RAxML.

4.2 Hypothesis testing and alternative topologies in the Bayesian posterior tree sample

347 In a hypothesis testing framework, the optimal topology from the *BEAST GT-ST framework is

348 a significantly worse fit compared to the concatenated ML best tree. Conversely the concatenated

349 ML best tree topology is absent from the 180,003 posterior trees produced in the *BEAST GT-

350 ST analysis. Combined, these demonstrate that strong conflicting signal underlies these

351 topological differences. Recent studies have alternatively suggested that concatenation may 
352 perform better than GT-ST when individual loci are not long enough to resolve phylogenies

353 (Gatesy and Springer, 2014), that concatenation and GT-ST methods should behave similarly

354 under a range of conditions (Tonini et al., 2015), and that phylogenomic scale data sets may

355 exacerbate problems of model misspecification (Liu et al., 2015). For additional discussion

356 around these issues see also Edwards et al., (2016) and Springer and Gatesy (2016). At present,

357 the relationships of the argentiniforms and stomatiids to neoteleosts remain unclear and may

358 depend strongly on the inclusion of the Galaxiidae. The placement of galaxiids has been unstable

359 (Betancur-R. et al., 2013; Burridge et al., 2012; Campbell et al., 2013; Ishiguro et al., 2003; Li et

360 al., 2010; López et al., 2004; Near et al., 2012), although independent studies e.g. (Campbell et

361 al., 2013; Near et al., 2012) suggest that galaxiids may be the sister lineage of the Neoteleostei.

362

3634.3 Lack of evidence for the monophyly of protacanthopterygians

364 The Protacanthopterygii is an historically important taxon of early-branching euteleosts with its

365 definition and content repeatedly modified (e.g. Greenwood et al., 1966; Johnson and Patterson,

366 1996; Lauder and Liem, 1983; Rosen, 1973; Rosen and Greenwood, 1970; Rosen and Patterson,

367 1969). Protacanthopterygian monophyly as defined by morphology (e.g. Johnson and Patterson,

368 1996) was questioned by molecular phylogenetics (Ishiguro et al., 2003). More recently, the

369 Protacanthopterygii was redefined by Betancur-R. et al. (2013) with molecular phylogenetics (bs

370 of $37 \%$ ) containing the Argentiniformes, Galaxiiformes, Esociformes and Salmoniformes.

371 Although we were unable to obtain representatives of Galaxiiformes, our analyses demonstrate

372 that the Argentiniformes are not most closely related to the Esociformes + Salmoniformes. A

373 topology test using available taxa in this dataset further rejected the Protacanthopterygii sensu

374 Betancur-R. et al. (2013). 


\section{5. Conclusions}

377 The two approaches (concatenation and GT-ST) implemented in this study indicate large areas of

378 congruence in topology resolving several relationships within early-branching euteleost

379 relationships. However, the disagreements highlight some of the potential caveats in resolving all

380 relationships of the early-branching euteleosts. We report the first study using a joint GT-ST

381 method to examine the question of early-branching euteleost relationships. A joint estimation of

382 species tree and gene trees can be preferred over summary methods (Gatesy and Springer, 2014)

383 and produced a slightly different hypothesis of relationships when compared to concatenated

384 analyses. A test of topology rejects the species-tree topology over the best scoring concatenated

385 ML topology. Likewise, posterior support for the *BEAST Bayesian species tree hypothesis is

386 high for early-branching euteleost nodes, indicating very few occurrences of alternative

387 topologies in the tree search. For major euteleost lineages, relationships among Argentiniformes,

388 Neoteleostei and Stomiatii differed in the results of concatenated ML and Bayesian joint GT-ST

389 analyses. This is in line with previous research on early-branching euteleost relationships. The

390 lack of agreement between studies of early-branching euteleost relationships may be caused by

391 short internode distances deep in the evolutionary past, leading to the formation and preservation

392 of few informative characters linking these old lineages. A related but less likely possibility is

393 that short internodes associated with very rapid diversification created conditions conducive to

394 pervasive ILS at the base of the euteleost radiation resulting in conflicting histories across

395 euteleost genomes and incongruent results between studies of early-branching euteleost

396 relationships. 
398 three areas of incongruence: 1) argentiniform sister lineage, 2) the placement of the

399 alepocephaliform lineage Bajacalifornia, and 3) the arrangement of the three neoteleost lineages

400 Antennarius, Acanthurus and Taenianotus. The percomorph taxa (Antennarius, Acanthurus and

401 Taenianotus) belong in a set of fish lineages whose relationship have been particularly difficult

402 to elucidate (Nelson, 1989). The incongruent inferences we observed between approaches may

403 be differential effects of ILS on coalescent versus non-coalescent phylogenetic approaches.

404 In terms of main early-branching euteleost lineages, only the placement of

405 Argentiniformes between concatenated and GT-ST hypotheses varied. The placement of the

406 argentiniform fishes is unresolved by this study and that branching between the Neotelostei,

407 Stomiati and Argentiniformes may be considered a soft polytomy. We find that phylogenomics

408 and the application of the coalescent model in phylogenetics strengthen support for the earliest

409 splits in the euteleostean radiation. However, key aspects of early euteleost phylogeny remain

410 unresolved and leave open the question of whether extant genomes from these lineages retain

411 historical signal that can be retrieved above the noise accumulated over hundreds of millions of

412 years of independent evolution.

\section{Acknowledgements}

415 We would like to acknowledge the following institutions and individuals that contributed to

416 sampling in this project: Burke Museum of Natural History and Culture, Kansas University

417 Biodiversity Institute and Natural History Museum, Academy of Natural Sciences of

418 Philadelphia, Robert Marcotte (Univeristy of Alaska Fairbanks), Molly Hallock (Washington

419 Department of Fish and Wildlife, retired), Motohiro Kikuchi (Chitose Salmon Park) and Peter 
420 Unmack (University of Canberra). Sébastien Lavoué (National Taiwan University), Thaddaeus

421 Buser (Oregon State University) and Kerry Reid (University of California Santa Cruz) provided

422 very helpful comments on drafts of the manuscript. We would like to thank Kevin McCracken

423 (University of Miami) for his support in promoting Next-Generation Sequencing and

424 Bioinformatics at the University of Alaska Fairbanks. 


\section{References}

426 Alfaro, M.E., Holder, M.T., 2006. The posterior and the prior in Bayesian phylogenetics. Annu. Rev. Ecol. Evol. Syst. 37, 19-42. doi:10.1146/annurev.ecolsys.37.091305.110021

428

429

430

431

432

434

435

436

437

438

439

440

441

442

443

444

445

446

447

Alfaro, M.E., Zoller, S., Lutzoni, F., 2003. Bayes or bootstrap? A simulation study comparing the performance of Bayesian Markov Chain Monte Carlo sampling and bootstrapping in assessing phylogenetic confidence. Mol. Biol. Evol. 20, 255-266. doi:10.1093/molbev/msg028

Allendorf, F., Thorgaard, G., 1984. Tetraploidy and the evolution of salmonid Fishes, in: Turner, B. (Ed.), Evolutionary Genetics of Fishes, Monographs in Evolutionary Biology. Springer US, pp. 1-53.

Betancur-R., R., Broughton, R.E., Wiley, E.O., Carpenter, K., López, J.A., Li, C., Holcroft, N.I., Arcila, D., Sanciangco, M., Cureton II, J.C., Zhang, F., Buser, T., Campbell, M.A., Ballesteros, J.A., Roa-Varon, A., Willis, S., Borden, W.C., Rowley, T., Reneau, P.C., Hough, D.J., Lu, G., Grande, T., Arratia, G., Ortí, G., 2013. The tree of life and a new classification of bony fishes. PLoS Curr, 5, 1-33. doi:10.1371/currents.tol.53ba26640df0ccaee75bb165c8c26288

Bolger, A.M., Lohse, M., Usadel, B., 2014. Trimmomatic: a flexible trimmer for Illumina sequence data. Bioinformatics 30, 2114-2120. doi:10.1093/bioinformatics/btu170

Burridge, C.P., McDowall, R.M., Craw, D., Wilson, M.V.H., Waters, J.M., 2012. Marine dispersal as a pre-requisite for Gondwanan vicariance among elements of the galaxiid fish fauna. J. Biogeogr. 39, 306-321. doi:10.1111/j.1365-2699.2011.02600.x

Campbell, M.A., López, J.A., Sado, T., Miya, M., 2013. Pike and salmon as sister taxa: Detailed intraclade resolution and divergence time estimation of Esociformes + Salmoniformes 
based on whole mitochondrial genome sequences. Gene 530, 57-65. doi:10.1016/j.gene.2013.07.068

Delsuc, F., Brinkmann, H., Philippe, H., 2005. Phylogenomics and the reconstruction of the tree of life. Nat. Rev. Genet. 6, 361-375. doi:10.1038/nrg1603

Drummond, A.J., Suchard, M.A., Xie, D., Rambaut, A., 2012. Bayesian phylogenetics with BEAUti and the BEAST 1.7. Mol. Biol. Evol. 29, 1969-73. doi:10.1093/molbev/mss075

Edwards, S.V., 2009. Is a new and general theory of molecular systematics emerging? Evolution

Edwards, S.V., Xi, Z., Janke, A., Faircloth, B.C., McCormack, J.E., Glenn, T.C., Zhong, B., Wu, S., Lemmon, E.M., Lemmon, A.R., Leaché, A.D., Liu, L., Davis, C.C., 2016. Implementing and testing the multispecies coalescent model: A valuable paradigm for phylogenomics. Mol. Phylogenet. Evol. 94, Part A, 447-462. doi:10.1016/j.ympev.2015.10.027

Faircloth, B.C., 2015. PHYLUCE is a software package for the analysis of conserved genomic

loci. Bioinformatics, btv646 doi:10.1093/bioinformatics/btv646

463

464

465

466

467

468

469

470 63, 1-19. doi:10.1111/j.1558-5646.2008.00549.x

Faircloth, B.C., Glenn, T.C., 2012. Not all sequence tags are created equal: designing and validating sequence identification tags robust to indels. PloS One 7, e42543.

Faircloth, B.C., McCormack, J.E., Crawford, N.G., Harvey, M.G., Brumfield, R.T., Glenn, T.C., 2012. Ultraconserved elements anchor thousands of genetic markers spanning multiple evolutionary timescales. Syst. Biol, sys004. doi:10.1093/sysbio/sys004

Faircloth, B.C., Sorenson, L., Santini, F., Alfaro, M.E., 2013. A phylogenomic perspective on the radiation of ray-finned fishes based upon targeted sequencing of ultraconserved elements (UCEs). PLoS ONE 8, e65923. doi:10.1371/journal.pone.0065923 
471 Felsenstein, J., 1978. Cases in which parsimony or compatibility methods will be positively 472 misleading. Syst. Biol. 27, 401-410. doi:10.1093/sysbio/27.4.401

473 Gatesy, J., Springer, M.S., 2014. Phylogenetic analysis at deep timescales: Unreliable gene trees, 474 475 bypassed hidden support, and the coalescence/concatalescence conundrum. Mol. Phylogenet. Evol. 80, 231-266. doi:10.1016/j.ympev.2014.08.013

476

477

478

479

480

481

482

483

484

485

486

487

488

489

490

491

492

493

Gilbert, P.S., Chang, J., Pan, C., Sobel, E.M., Sinsheimer, J.S., Faircloth, B.C., Alfaro, M.E., 2015. Genome-wide ultraconserved elements exhibit higher phylogenetic informativeness than traditional gene markers in percomorph fishes. Mol. Phylogenet. Evol. 92, 140-146. doi:10.1016/j.ympev.2015.05.027

Greenwood, P.H., Myers, G.S., Rosen, D.E., Weitzman, S.I.I., 1967. Named main divisions of teleostean fishes. Proc. Biol. Soc. Wash. 80, 227-228.

Greenwood, P.H., Rosen, D.E., Weitzman, S.H., Myers, G.S., 1966. Phyletic studies of teleostean fishes, with a provisional classification of living forms. Bull. Am. Mus. Nat. Hist. 131, 339-456.

Harrington R.C., Faircloth B.C., Eytan R.I., Smith W.L., Near T.J., Alfaro M.E., Friedman M., 2016. Phylogenomic analysis of carangimorph fishes reveals flatfish asymmetry arose in a blink of the evolutionary eye. BMC Evol. Biol. 16, 224. doi: 10.1186/s12862-0160786-x

Heled, J., Drummond, A.J., 2010. Bayesian Inference of Species Trees from Multilocus Data. Mol. Biol. Evol. 27, 570-580. doi:10.1093/molbev/msp274

Hillis, D.M., Bull, J.J., 1993. An empirical test of bootstrapping as a method for assessing confidence in phylogenetic analysis. Syst. Biol. 42, 182-192.

doi:10.1093/sysbio/42.2.182 
494 Huelsenbeck, J.P., 1997. Is the Felsenstein Zone a fly trap? Syst. Biol. 46, 69-74.

495 doi:10.1093/sysbio/46.1.69

496 Inoue, J.G., Miya, M., Tsukamoto, K., Nishida, M., 2003. Basal actinopterygian relationships: a 497 mitogenomic perspective on the phylogeny of the "ancient fish." Mol. Phylogenet. Evol.

498

499

500

501

502

503

504

505

506

507

508

509

510

511

512

513

514 26, 110-120. doi: 10.1016/S1055-7903(02)00331-7

Ishiguro, N.B., Miya, M., Nishida, M., 2003. Basal euteleostean relationships: a mitogenomic perspective on the phylogenetic reality of the "Protacanthopterygii." Mol. Phylogenet. Evol. 27, 476-488. doi: 10.1016/S1055-7903(02)00418-9

Johnson, G.D., Patterson, C., 1996. Relationships of lower euteloestean fishes, in: Stiassny, M.L.J. (Ed.), Interrelationships of Fishes. Academic Press, pp. 251-328.

Katoh, K., Misawa, K., Kuma, K., Miyata, T., 2002. MAFFT: a novel method for rapid multiple sequence alignment based on fast Fourier transform. Nucleic Acids Res. 30, 3059 -3066. doi:10.1093/nar/gkf436

Knowles, L.L., Kubatko, L.S., 2011. Estimating species trees: practical and theoretical aspects. John Wiley and Sons.

Lauder, G., Liem, K., 1983. The evolution and interrelationships of the actinopterygian fishes. Bull. Mus. Comp. Zool. 150, 95-197.

Lavoué, S., Miya, M., Poulsen, J.Y., Moller, P.R., Nishida, M., 2008. Monophyly, phylogenetic position and inter-familial relationships of the Alepocephaliformes (Teleostei) based on whole mitogenome sequences. Mol. Phylogenet. Evol. 47, 1111-1121. doi:

10.1016/j.ympev.2007.12.002 
515 Lemmon, E.M., Lemmon, A.R., 2013. High-throughput genomic data in systematics and

516 phylogenetics. Annu. Rev. Ecol. Evol. Syst. 44, 99-121. doi: 10.1146/annurev-ecolsys-

517 $110512-135822$

518 Li, J., Xia, R., McDowall, R.M., López, J.A., Lei, G., Fu, C., 2010. Phylogenetic position of the enigmatic Lepidogalaxias salamandroides with comment on the orders of lower euteleostean fishes. Mol. Phylogenet. Evol. 57, 932-936.

521 doi:10.1016/j.ympev.2010.07.016

Liu, L., Yu, L., Pearl, D.K., Edwards, S.V., 2009. Estimating species phylogenies using coalescence times among sequences. Syst. Biol. 58, 468-477. doi: 10.1093/sysbio/syp031

524 Liu, L., Yu, L., Edwards, S.V., 2010. A maximum pseudo-likelihood approach for estimating species trees under the coalescent model. BMC Evol. Biol. 10, 302. doi: 10.1186/14712148-10-302

Liu, L.,Yu, L., 2011. Estimating species trees from unrooted gene trees. Syst. Biol. 60, 661-667. doi: $10.1093 /$ sysbio/syr027

Liu, L., Xi, Z., Wu, S., Davis, C.C., Edwards, S.V., 2015. Estimating phylogenetic trees from genome-scale data. Ann. N. Y. Acad. Sci. 1360, 36-53. doi:10.1111/nyas.12747

531 López, J.A., Chen, W.J., Ortí, G., 2004. Esociform phylogeny. Copeia 2004, 449-464. doi: 10.1643/CG-03-087R1

533 Mirarab, S., Reaz, R., Bayzid, M.S., Zimmermann, T., Swenson, M.S., Warnow, T., 2014. ASTRAL: genome-scale coalescent-based species tree estimation. Bioinformatics, 30, i541-i548. doi:10.1093/bioinformatics/btu462 
536 Mirarab, S., Warnow, T., 2015. ASTRAL-II: coalescent-based species tree estimation with many

537 hundreds of taxa and thousands of genes. Bioinformatics, 31 44-i52.

538 doi:10.1093/bioinformatics/btv234

539 Moravec, J., 2015. BayesMonophyly. Available at https://github.com/J-

$540 \quad$ Moravec/BayesMonophyly

541 Near, T.J., Eytan, R.I., Dornburg, A., Kuhn, K.L., Moore, J.A., Davis, M.P., Wainwright, P.C.,

542 Friedman, M., Smith, W.L., 2012. Resolution of ray-finned fish phylogeny and timing of

543 diversification. Proc. Natl. Acad. Sci. 109, 13698-13703. doi:10.1073/pnas.1206625109

544 Nelson, G., 1989. Phylogeny of major fish groups, in: Fernholm, B., Bremer, K., Brundin, L.,

545 Jornvall, H., Rutberg, L., Wanntorp, H.-E. (Eds.), The Hierarchy of Life. Elsevier,

$546 \quad$ Amsterdam, pp. 325-336.

547 Nelson, J.S., 2006. Fishes of the world, 4th ed. John Wiley and Sons, Inc., New York.

548 Pyron, R.A., 2015. Post-molecular systematics and the future of phylogenetics. Trends Ecol.

$549 \quad$ Evol. 30, 384-389. doi:10.1016/j.tree.2015.04.016

550 Rokas, A., Carroll, S.B., 2006. Bushes in the tree of life. PLoS Biol. 4, e352.

551 Rosen, D., 1973. Interrelationships of higher teleostean fishes, in: Greenwood, P., Miles, R.,

552 Patterson, C. (Eds.), Interrelationships of Fishes. Academic Press, London, pp. 397-513.

553 Rosen, D.E., 1985. An essay on euteleostean classification. Am. Mus. Novit. 2827, 1-57.

554 Rosen, D., Greenwood, P., 1970. Origin of the Weberian apparatus and the relationships of the

555 ostariophysan and gonorynchiform fishes. Am. Mus. Novit. 2428, 1-25.

556 Rosen, D., Patterson, C., 1969. The structure and relationships of the paracanthopterygian fishes. Bull. Am. Mus. Nat. Hist. 141, 357-474. 
558 Salichos, L., Rokas, A., 2013. Inferring ancient divergences requires genes with strong

559 phylogenetic signals. Nature 497, 327-331.

560 Shaw, T.I., Ruan, Z., Glenn, T.C., Liu, L., 2013. STRAW: species tree analysis web server.

$561 \quad$ Nucleic Acids Res, gkt377. doi: $10.1093 /$ nar/gkt377

562 Shen, X.X., Hittinger, C.T., Rokas, A., 2017. Contentious relationships in phylogenomic studies 563 can be driven by a handful of genes. Nature Ecol. Evol. 1, 0126. doi:10.1038/s41559-

564 017-0126

Shimodaira, H., Hasegawa, M., 2001. CONSEL: for assessing the confidence of phylogenetic tree selection. Bioinformatics 17, 1246-1247. doi:10.1093/bioinformatics/17.12.1246

Springer, M.S., Gatesy, J., 2016. The gene tree delusion. Mol. Phylogenet. Evol. 94, Part A, 133. doi:10.1016/j.ympev.2015.07.018

Stamatakis, A., 2014. RAxML version 8: a tool for phylogenetic analysis and post-analysis of large phylogenies. Bioinformatics 30, 1312-1313. doi:10.1093/bioinformatics/btu033

571

572

573

574

575

576

577

578

579

Suzuki, Y., Glazko, G.V., Nei, M., 2002. Overcredibility of molecular phylogenies obtained by Bayesian phylogenetics. Proc. Natl. Acad. Sci. 99, 16138-16143. doi:10.1073/pnas.212646199

Tonini, J., Moore, A., Stern, D., Shcheglovitova, M., Ortí, G., 2015. Concatenation and species tree methods exhibit statistically indistinguishable accuracy under a range of simulated conditions. PLoS Curr. 7, ecurrents.tol.34260cc27551a527b124ec5f6334b6be. doi:10.1371/currents.tol.34260cc27551a527b124ec5f6334b6be

Wilson, M.V.H., Williams, R.R.G., 2010. Salmoniform fishes: key fossils, supertree, and possible morphological synapomorphies., in: Nelson, J.S., Schultze, H.P., Wilson, 
580 M.V.H. (Eds.), Origin and Phylogenetic Interrelationships of Teleosts. Verlag Dr.

$581 \quad$ Friedrich Pfeil, München, pp. 379-409.

582 Zerbino, D.R., Birney, E., 2008. Velvet: Algorithms for de novo short read assembly using de

583 Bruijn graphs. Genome Res. 18, 821-829. doi:10.1101/gr.074492.107 